\title{
La pitaya (Hylocereus sp.) en culture de diversification à l'île de la Réunion : stratégie d'accompagnement de son développement
}

Fabrice Le Bellec ${ }^{a \star}$, René-Claude Judith ${ }^{\mathrm{b}}$

a Cirad-Flhor,

Station de Neufchâteau, 97130 Capesterre B/E,

Guadeloupe, France

b Cirad-Flhor,

BP 180,

97455 Saint-Pierre Cedex, île de La Réunion, France

*Correspondance et tirés à part

Reçu le 20 novembre 2001 Accepté le 25 mars 2002

Fruits, 2002, vol. 57, p. 219-230 (c) 2002 Cirad/EDP Sciences All rights reserved DOI: 10.1051/fruits:2002019

RESUMEN Español, p. 230

\section{The pitaya (Hylocereus sp.) as a diversification crop in Réunion: strategy of its development accompaniment.}

Abstract - Fruit-bearing plant heritage of Réunion. Among nearly 138 fruit-bearing species which make up the fruit plant heritage of Réunion, only some are actually cultivated. However, some others could attract avid consumers of new products. Criteria of preselection for a diversification crop. Considering this report, a preselection was carried out in order to identify new fruit-bearing species able to be used as a diversification crop in Réunion; the selection criteria were: the attractiveness of the fruit, its convenience for being consumed, its taste, the conditions for its storage, the profitability of the species, etc. The pitaya (Hylocereus sp.) was then retained. This choice was then validated by a fruit consumption survey on Réunion consumers. Development of the pitaya in Réunion. The development of this new crop was carried out in three stages: identification of the agronomic constraints related to the intensification of this cultivation and the search for solutions; acquisition of the basic farming techniques and sensitizing of the general public; the taking into account for the farmers' constraints, and commercial accompaniment for the new product. Conclusions. The commercial quality of the pitaya due to its attractive color and shape, the consumers' enthusiasm and the farmers' motivation towards this new species make $H$. sp. a real opportunity for diversification for Réunion whereas, 7 years ago, the fruit of this species was completely unknown on the island.

Réunion / Hylocereus / diversification / agricultural development / crop management / farmers / merchants / consumer surveys

La pitaya (Hylocereus sp.) en culture de diversification à l'île de la Réunion : stratégie d'accompagnement de son développement.

Résumé — Le patrimoine fruitier de l'île de la Réunion. Parmi les quelques 138 espèces fruitières qui composent le patrimoine fruitier de l'île de la Réunion, seules quelques-unes d'entre-elles sont véritablement cultivées. Pourtant, certaines autres pourraient séduire les consommateurs avides de nouveauté. Critères de présélection pour une culture de diversification. Face à ce constat, une présélection a été réalisée afin d'identifier des espèces fruitières nouvelles, candidates à la diversification pour l'île de la Réunion ; les critères de choix ont été : l'attractivité du fruit, sa commodité à être consommé, son goût, l'aptitude à sa conservation, la rentabilité de l'espèce, etc. La pitaya (Hylocereus sp.) a alors été retenue. Ce choix a ensuite été validé par une enquête auprès de consommateurs réunionnais. Développement de la pitaya à l'île de la Réunion. Le développement de cette nouvelle culture a été réalisé en trois étapes : identification des contraintes agronomiques liées à l'intensification de cette culture et recherche de solutions ; acquisition des techniques culturales de base et sensibilisation du grand public ; prise en compte des contraintes des agriculteurs et accompagnement commercial du nouveau produit. Conclusions. Les qualités commerciales de la pitaya (couleur et forme attrayantes), l'enthousiasme des consommateurs et la motivation des agriculteurs envers cette nouvelle espèce font de $H$. sp. une véritable opportunité de diversification pour l'île de la Réunion alors que, il y a 7 ans, le fruit de cette espèce était totalement inconnu sur l'île.

Réunion / Hylocereus / diversification / développement agricole / conduite de la culture / agriculteur / négociant / enquête auprès des consommateurs 


\section{Le patrimoine fruitier de l'île de la Réunion}

Presque toutes les espèces fruitières présentes aujourd'hui à l'île de la Réunion y ont été introduites. Les premières introductions ont débuté dès la fin $\mathrm{XvI}^{\mathrm{e}}$ siècle $[1,2]$ et d'autres ont suivi jusqu'à nos jours. Un inventaire récent a permis de répertorier près de 138 espèces fruitières différentes sur l'île [3]. Si ce patrimoine peut sembler conséquent, toutes ces espèces n'ont pas pour autant aujourd'hui la même importance. Trois groupes ont pu être identifiés : les espèces cultivées à des fins commerciales, les espèces naturalisées et/ou acclimatées et, enfin, les autres espèces de jardins peu ou pas développées.

Le premier groupe est représenté par cinq productions principales : l'ananas, la mangue, le litchi, la banane et les agrumes. Jusqu'ici considérées comme des cultures d'appoint, ces productions s'apparentent aujourd'hui à de véritables filières.

Les espèces naturalisées et acclimatées représentent quant à elles près d'un quart du patrimoine. Leurs fruits peuvent jouer un rôle non négligeable sur les marchés ; c'est le cas, par exemple, du goyavier-fraise (Psidium cattleianum), du néflier du Japon (Eriobotrya japonica), du jacquier (Artocarpus heterophyllus), du longani (Nephelium longan) ou de la pomme cannelle (Annona squamosa). Leur approvisionnement est cependant difficilement contrôlable car, bien souvent, il provient de cueillettes. Ces espèces naturalisées sont aussi parfois devenues envahissantes vis-àvis des plantes indigènes [4] ; c'est le cas notamment de la vigne marronne (Rubus alcefolius), du jamrosade (Sysygium jambos) ou encore du goyavier-fraise (Psidium cattleianum).

Le reste du patrimoine fruitier, qui en représente la plus grande part, correspond aux espèces cultivées dans les jardins. Depuis une quinzaine d'années, les collectivités réunionnaises ont consenti un effort certain pour réhabiliter l'ensemble de ce patrimoine par le financement de plantations. De plus, l'essor touristique que connaît l'île de la Réunion depuis quelques années incite les agriculteurs à promouvoir une agriculture de service, notamment par le développement des fermes/auberges. Le verger créole prend alors toute son importance et devrait permettre de valoriser une partie de la diversité du patrimoine fruitier soit par une consommation directe du producteur au consommateur (fermes/auberges), soit par la mise en marché d'espèces fruitières, populaires ou non, jusqu'alors peu ou pas cultivées.

Dans ce contexte, une démarche a été adoptée à l'île de la Réunion pour sélectionner, développer et accompagner une nouvelle espèce fruitière, la pitaya ( $\mathrm{Hylo}$ cereus sp.). La synthèse présentée explique pourquoi l'espèce a été sélectionnée parmi une vingtaine d'autres, quand et comment le producteur a pu être impliqué pour son développement et quelles ont été les démarches d'accompagnement technique et commercial de cette culture fruitière jusqu'alors inconnue dans l'île. La prise en compte de ces différents aspects de la démarche est apte à conditionner étroitement la réussite d'une opération de diversification car les causes d'échecs et de succès de telles expériences peuvent être nombreuses [5-8] : nature des débouchés commerciaux, relation et compétition entre les cultures pivots et cultures de diversification, acquisition des compétences culturales par le producteur, problème d'adéquation au marché, adaptation écologique de l'espèce, problèmes agronomiques, etc. En effet, le lancement d'une culture nouvelle n'est pas sans contraintes et leur anticipation grâce à une approche pluridisciplinaire est probablement la clé d'une diversification réussie.

\section{Critères de présélection}

Partant du constat qu'il existait déjà, à l'île de la Réunion, une grande diversité de fruits de formes, goûts et couleurs différents, il n'était donc pas nécessaire d'introduire un hypothétique nouveau fruit, mais bien d'évaluer les potentialités de certains fruits sous-utilisés ; ces espèces présentaient en outre l'avantage d'être déjà acclimatées aux conditions pédoclimatiques de l'île. 
Un premier criblage du patrimoine fruitier réunionnais a permis d'identifier près d'une centaine d'espèces manifestement insuffisamment développées dans l'île. Une présélection a ensuite été réalisée afin d'identifier des espèces fruitières nouvelles candidates à la diversification [9]

Une approche basée sur les exigences des divers acteurs de la filière a été retenue. Elle s'est nécessairement basée en priorité sur les critères de choix du consommateur (tableau I) avant d'être affinée par la prise en compte des critères du vendeur puis du producteur (tableau II). Ainsi ont été considérés :

- pour le consommateur, l'attractivité du nouveau fruit évaluée au travers de sa forme, de sa couleur, de son goût (bon équilibre sucre/acidité, aptitude à désaltérer, etc.) et de sa facilité à être consommé ;

- pour le vendeur, l'intérêt du nouveau fruit au travers de sa durée de vie sur les rayons, de ses possibilités de conservation et de la régularité de son approvisionnement (soit tout au long de l'année, soit saisonnier). Si des solutions techniques peuvent être envisagées pour pallier d'éventuelles lacunes à ce niveau, elles sont coûteuses ;

- pour l'agriculteur, la rentabilité de la nouvelle culture assurée par une culture aisée et une mise à fruit rapide (retour sur investissement). C'est en fait à ce producteur que reviendra finalement la décision de développer ou pas une nouvelle espèce, en se guidant sur la possibilité d'intégrer cette culture dans son système d'exploitation et sur la capacité de l'espèce à s'adapter à son environnement.

Après une présélection basée par anticipation sur les exigences émanant à la fois du consommateur, du vendeur et de l'agriculteur, les choix devront être ensuite confrontés et validés par le grand public.

En s'appuyant sur de telles considérations, 21 espèces présentes à l'île de la Réunion (tableau II) auraient pu séduire le consommateur, mais seulement huit d'entre elles ayant atteint une note finale supérieure ou égale à 12 se sont révélées susceptibles de satisfaire également les exigences du vendeur et du producteur : Cyphomandra betacea (tamarillo), Diospyros kaki (kaki), Fortunella sp. (kumquat), Hovenia dulcis (raisin de Chine), Hylocereus sp. (pitaya), Manilkara zapota (sapotillier), Passiflora ligularis (grenadelle) et Physalis peruviana (physalis).

Finalement, comme il semblait difficile d'accompagner le développement de ces huit espèces de front, seule l'une d'entreelles a été retenue : la pitaya (figure 1). La forme nouvelle du fruit, ses couleurs attrayantes, la rapidité de mise à fruit de la plante et les bonnes aptitudes de conservation du fruit ont motivé cette sélection.

Tableau I.

Schématisation de la méthode utilisée à l'île de la Réunion pour présélectionner les espèces fruitières intéressantes à développer dans le cadre d'une démarche de diversification à partir des critères propres au consommateur.

\begin{tabular}{|c|c|c|c|}
\hline Étape & Patrimoine fruitier considéré & Critère appliqué & Espèces fruitières retirées \\
\hline $1^{\text {er }}$ criblage & 138 espèces acclimatées & $\begin{array}{l}\text { Espèces déjà exploitées } \\
\text { dites « espèces économiques » }\end{array}$ & Manguier, papayer, oranger, etc. \\
\hline $2^{e}$ criblage & 100 espèces sous-utilisées & $\begin{array}{l}\text { Note }^{1} \text { du consommateur } \\
\text { sur trois caractères : attractivité, } \\
\text { goût, facilité de consommation }\end{array}$ & $\begin{array}{l}\text { Toutes espèces dont la note globale } \\
\text { est inférieure à } 5 \text { (voir tableau II). }\end{array}$ \\
\hline
\end{tabular}


F. Le Bellec, R.-C. Judith

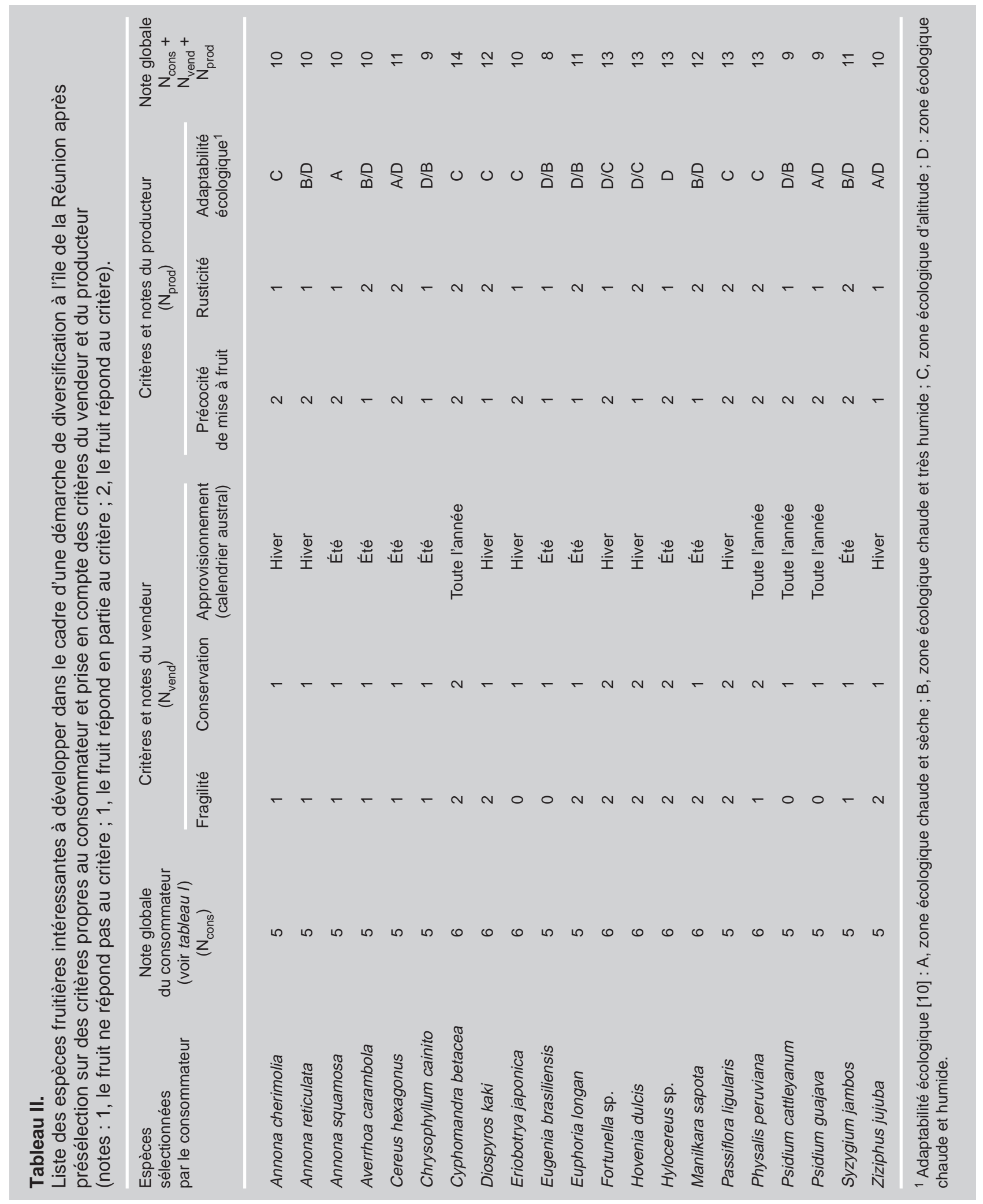




\section{La pitaya à l'île de la Réunion}

Originaire d'Amérique latine, la pitaya (genre Hylocereus) n'est cultivée à grande échelle que dans certains pays (Colombie, Costa Rica, Nicaragua et Vietnam). Ailleurs, la plante est considérée comme une culture nouvelle prometteuse [11-13].

L'introduction d'Hylocereus undatus Britt. \& Rose, la pitaya à chair blanche, sur l'île de la Réunion est ancienne ; l'espèce y est aujourd'hui naturalisée sur tout le littoral [14] (figure 2). La plante est populaire : elle sert de nourriture aux tortues terrestres et les racines sont utilisées en médecine traditionnelle [15]. Une autre pitaya, Hylocereus purpusii Britt. \& Rose à chair rouge, est également répertoriée sur l'île bien que moins représentée.

Les fruits de ces deux espèces étaient quant à eux inconnus des réunionnais [16], la production naturelle de fruits y étant quasi inexistante. L'auto-incompatibilité [17] des clones des deux espèces présentes, conjuguée à une absence de pollinisateurs efficaces, semblent être responsables de cette improductivité.

Alors qu'en 1994, la pitaya était un fruit inconnu à l'île de la Réunion, en 2001, près de 3 ha ont été implantés (10000 plants) impliquant la participation d'une vingtaine d'agriculteurs. En 2002, une dizaine de tonnes de fruits est attendue sur le marché. Ce résultat est la conséquence de la stratégie qui a été adoptée pour accompagner le développement de l'espèce et préparer le consommateur.

\section{Accompagnement et évaluation du développement de la pitaya à la Réunion}

Le statut de la plante sur l'île (espèce populaire mais fruit inconnu), les caractéristiques de stérilité de ses clones et les connaissances techniques insuffisantes ont influencé la stratégie utilisée. Le processus s'est appuyé sur trois phases qui ont été évaluées à partir de la définition d'indicateurs de résultat.

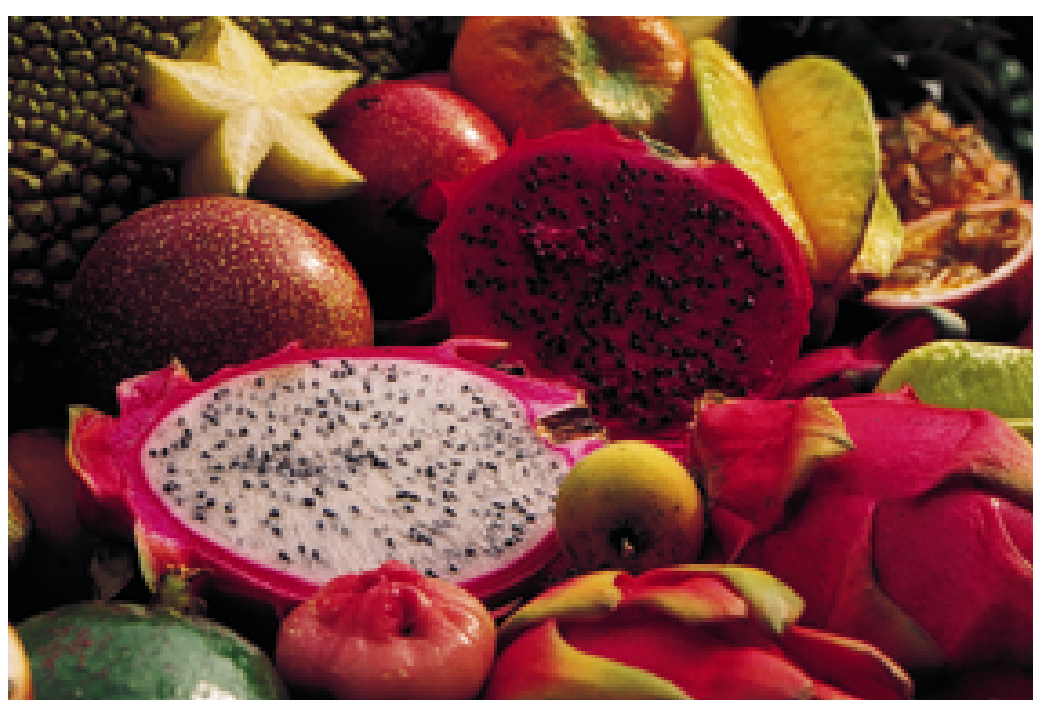

Figure 1.

Fruit d'Hylocereus undatus et de H. purpusii.

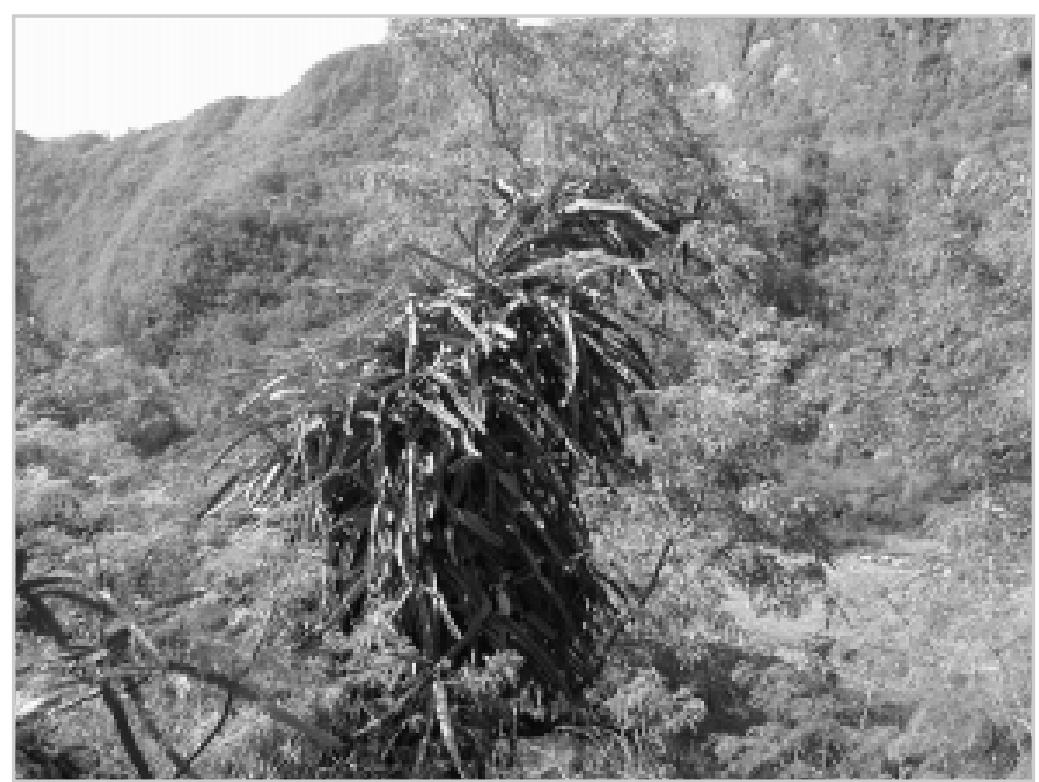

\subsection{Recherche en microparcelles et validation du choix de l'espèce}

Figure 2.

Naturalisation d'Hylocereus undatus à l'île de la Réunion.
Cette première phase s'est déroulée de 1994 à 1995. Pour pallier les problèmes de stérilité rencontrés dans la nature et pour évaluer les contraintes agronomiques liées à l'intensification de Hylocereus sp., nouvelle espèce à développer, une meilleure 


\begin{tabular}{|c|c|c|c|c|}
\hline \multirow[t]{2}{*}{ Question posée } & \multirow{2}{*}{$\begin{array}{l}\text { Question } \\
\text { ouverte }\end{array}$} & \multicolumn{2}{|c|}{ Questions fermées (\%) } & \multirow{2}{*}{$\begin{array}{l}\text { Sans avis } \\
\text { (\%) }\end{array}$} \\
\hline & & Oui & Non & \\
\hline Pensez-vous que ce fruit a un avenir commercial? & Sans objet & 82 & 3,5 & 14,5 \\
\hline $\begin{array}{l}\text { À quel prix pensez-vous qu'il se vendrait sur les marchés } \\
\text { (fruit présenté de } 400 \mathrm{~g} \text { ) ? }\end{array}$ & $1,51 €$ & Sans objet & Sans objet & 25,0 \\
\hline À quel prix maximum achèteriez-vous ce fruit? & $1,60 €$ & Sans objet & Sans objet & 17,5 \\
\hline $\begin{array}{l}\text { Quel prix de vente minimum vous ferait craindre une mauvaise } \\
\text { qualité de fruit? }\end{array}$ & $0,67 €$ & Sans objet & Sans objet & 36,5 \\
\hline Ce fruit vous fait-il penser à un autre fruit? & Kiwi & 76 & 24 & Sans objet \\
\hline
\end{tabular}

Figure 3.

Évolution des intentions d'achat au fur et à mesure de la découverte de la pitaya par des consommateurs interrogés à l'occasion d'une enquête effectuée à l'île de la Réunion. connaissance de la plante était indispensable. Pour cela, un travail sur microparcelles en station de recherche et la sélection de clones dans la nature ont été entrepris ; ils ont permis de surmonter les obstacles à la production. Il est alors devenu indispensable de vérifier auprès du grand public son éventuelle adhésion à ce nouveau fruit.

Cet aspect de la démarche de diversification fruitière a été traité à l'aide d'une enquête effectuée sur le marché forain de la ville du Tampon. Le lieu - rendez-vous hebdomadaire du Sud de la Réunion - et le créneau horaire - 13:00 h à 18:30 h - ont permis d'interroger 200 personnes se présentant d'elles-mêmes au stand. Celles-ci ont répondu à 30 questions au total, établies

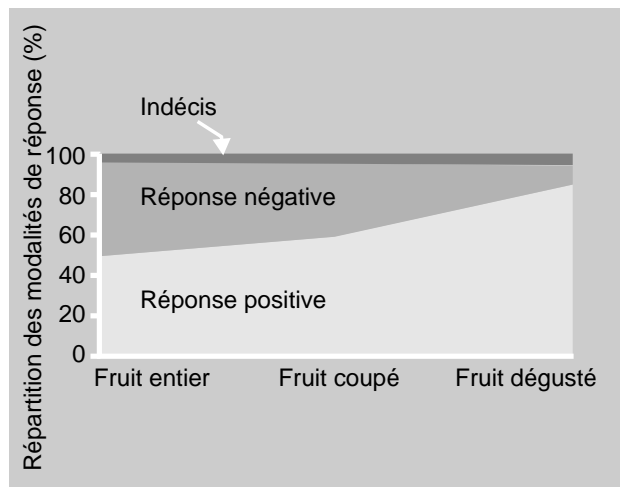

selon une chronologie logique : appréciation du fruit entier, puis du fruit coupé en deux ; appréciation sur dégustation ; enfin, intention d'achat et marge de prix acceptable (tableau III). Les deux variétés de pitaya, à chair blanche et à chair rouge, ont été présentées. La population enquêtée a été composée de $61,5 \%$ de femmes de tout âge, une majorité d'entre elles appartenant, cependant, à la tranche de 26 à 45 ans $(56,5 \%)$; la plupart des personnes interrogées $(87,5 \%)$ résidait sur l'île de la Réunion, les autres étant en séjour de vacances.

Pour la majorité (82\%) des personnes interrogées, la pitaya aurait un avenir commercial (tableau III). Cette opinion a été confirmée par le pourcentage final des consommateurs prêts à l'achat. Le nombre d'acheteurs potentiels a évolué au fur et à mesure de la découverte du produit (figure 3) ; après dégustation, 85,5\% des personnes enquêtées achèteraient la pitaya à un prix accepté de l'ordre de $10 \mathrm{FF}(1,51 €)$ l'unité. Ces résultats encourageants ont motivé la poursuite du développement de cette nouvelle espèce fruitière.

\subsection{Recherches en macroparcelles et sensibilisation}

Cette nouvelle étape qui a duré de 1996 à 1999 a permis de mettre au point un 
itinéraire technique de base à appliquer à la culture de la pitaya (tableau IV). Elle s'est déroulée en macroparcelles expérimentales sur station de recherche. Une fois validées, les données ont été transmises aux agriculteurs par le biais de formations destinées aux agents du développement agricole et aux producteurs. Ainsi, deux formations ont été organisées avec la Chambre d'agriculture et environ une centaine de producteurs ont été formés. La sensibilisation du grand public a été parallèlement entreprise par le biais de différents médias comme la presse écrite (quatre articles publiés dans la presse locale) et la télévision (deux reportages). L'opération a été nominée dans un concours organisé par la jeune Chambre économique de la Réunion (Réunion Bio).

\subsection{Recherche appliquée et accompagnement commercial}

Cette phase de recherche appliquée, commencée en 2000, s'est effectuée en milieu réel chez le producteur et en station de recherche. Elle a permis de prendre en compte les contraintes des agriculteurs, notamment par l'adaptation de l'itinéraire technique à l'exploitation. Parallèlement, l'accompagnement à la commercialisation du nouveau produit a été engagé : édition d'une fiche produit, dégustation organisée dans les foires, salons et hôtel (article de presse), suivi de la mise en marché forain et étude des débouchés en fruit frais, transformé ou pour la décoration. La légalisation commerciale du produit a été abordée par une participation à un projet de norme Codex de la DGCCRF (Direction générale de la concurrence, de la consommation et de la répression des fraudes).

À la suite de cet ensemble de mesures, les surfaces plantées et le nombre de producteurs concernés ont augmenté. Au travers de foires et de salons, il a été possible de se rendre compte de la perception positive du produit par le public, acceptation également constatée de la part de l'industriel (confection de sorbet), et de l'artisan (glacier et restaurateur). Le prix de vente fixé après enquête s'est révélé être bien accepté par le public, et la clientèle s'est fidélisée.

\section{La pitaya chez le producteur}

L'itinéraire technique de production de la pitaya à l'île de la Réunion ayant été mis au point à l'issue des différentes phases de la démarche de diversification entreprise, les agriculteurs concernés par le projet se sont facilement appropriés ces techniques culturales de base. La troisième phase de ce programme a alors permis d'adapter cet itinéraire à l'exploitation. Des solutions individuelles de type palissage de la plante (poteau individuel (figure 4), table de production (figure 5), pan incliné de production, etc.) ont été notamment recherchées afin de diminuer le coût de plantation élevé et d'assurer à l'agriculteur un retour sur investissement plus rapide (tableau $V$ ).

Aucune aide directe financière n'a été apportée aux agriculteurs concernés, sauf la fourniture du matériel végétal (boutures des trois variétés devant composer le verger). Tous les agriculteurs se sont entièrement autofinancés. Il en a résulté une parfaite intégration de cette nouvelle culture à leur système d'exploitation : surfaces développées réduites [entre (2000 et 5000) $\mathrm{m}^{2}$ ] et proportionnelles à leur exploitation, adaptation de l'itinéraire technique (choix du tuteur, des densités), valorisation de terrains difficiles, etc. Dans ces conditions, la
Figure 4.

Pitaya conduite sur tuteur individuel en bois en macroparcelles expérimentales à l'île de la Réunion.

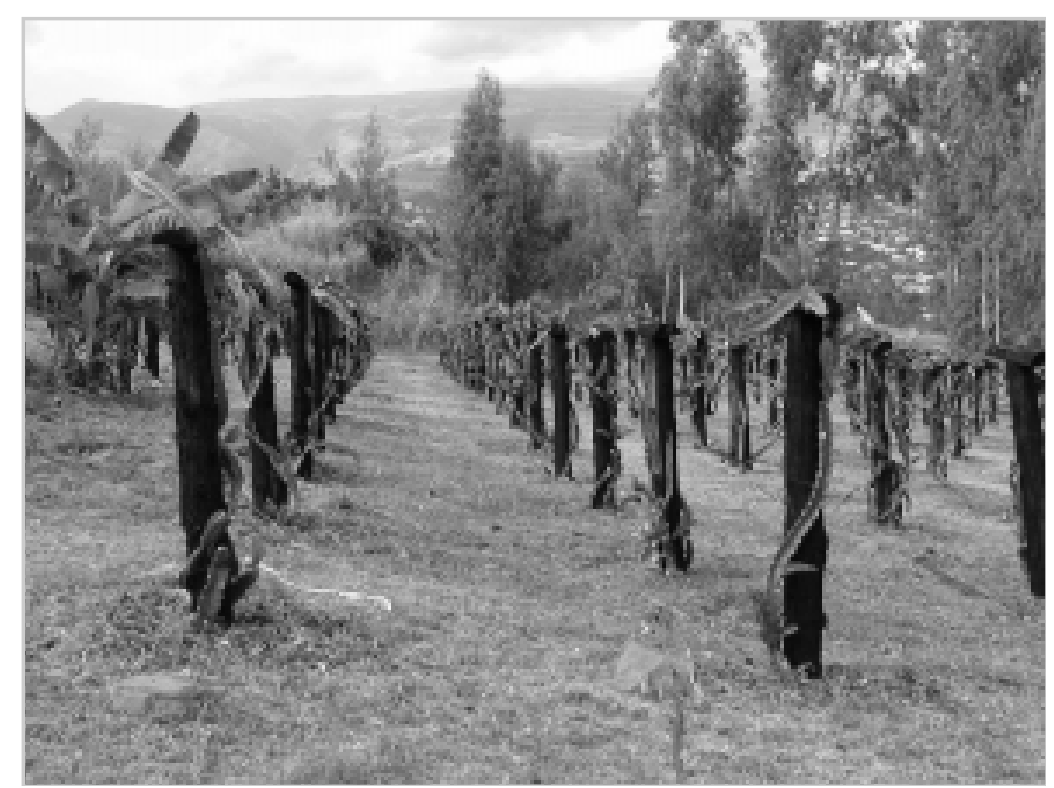




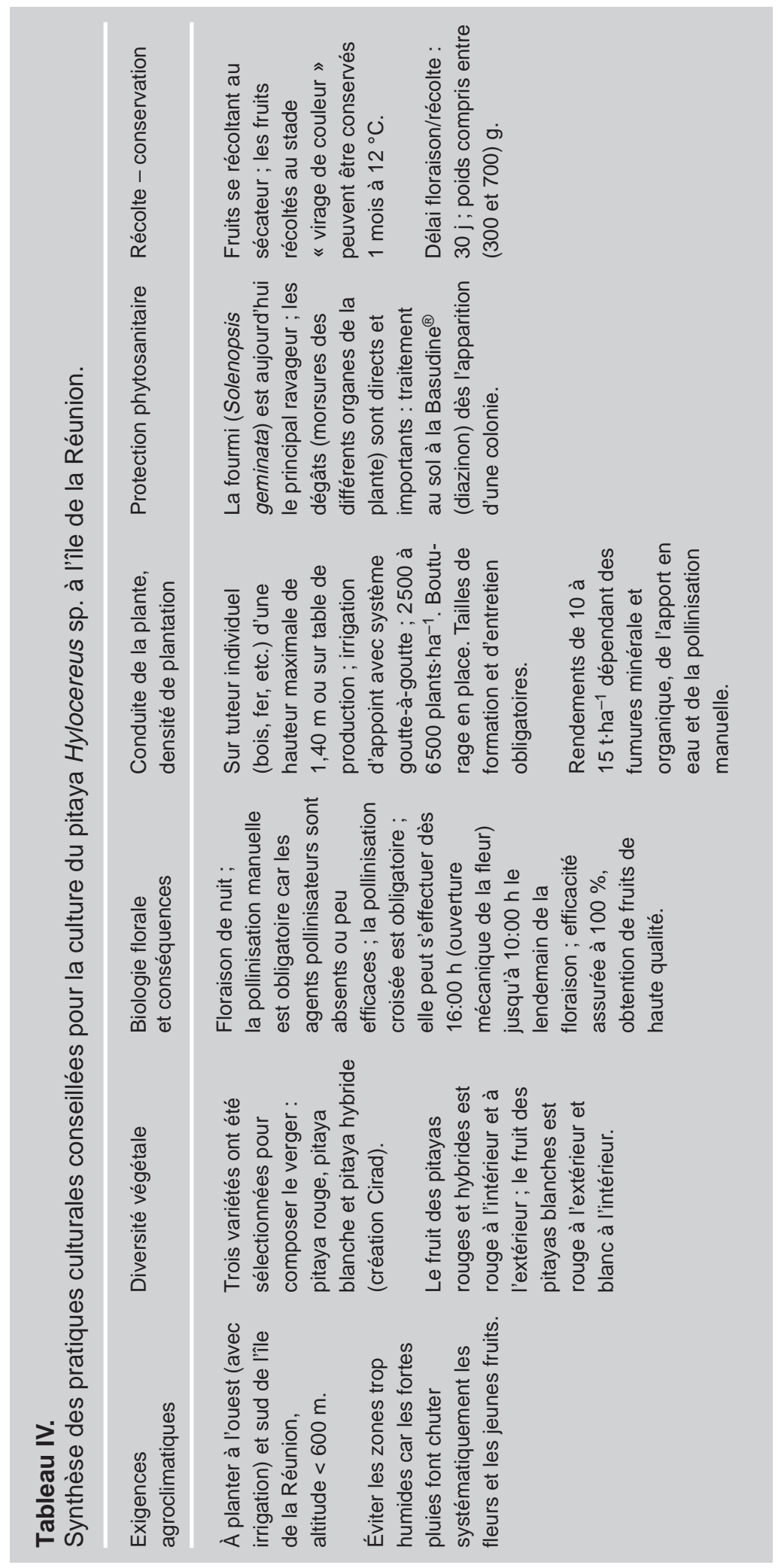


culture de la pitaya peut être maintenant considérée comme une culture de diversification complémentaire à une spéculation principale (canne à sucre, principalement). D'ailleurs, les différentes phases de ces deux cultures peuvent se coordonner particulièrement bien car les pics de travail ne se chevauchent pas (pollinisation-récolte de la pitaya de décembre à juin et coupe de la canne à sucre de juillet à décembre).

\section{Les débouchés de la pitaya}

La pitaya est maintenant accueillie très favorablement par le consommateur réunionnais [12] qui a été séduit tant par la forme, la couleur et la chair ferme et juteuse de ce fruit que par sa facilité à être consommé (figure 0). Le prix d'achat accepté est de $4,57 € \cdot \mathrm{kg}^{-1}$.

Le commerçant, quant à lui, apprécie certaines caractéristiques commerciales de la pitaya : fruit facile à conserver, peu fragile aux manipulations et dont la production est étalée sur 6 mois. Si la vente en frais est aujourd'hui à privilégier car la marge est alors plus intéressante pour le producteur, d'autres débouchés ne sont cependant pas à négliger. En effet, différents corps de métier s'intéressent à cette production : l'industrie agroalimentaire (sorbet, colorant naturel, etc.), ou la restauration (décoration, coupe de fruit, etc.).

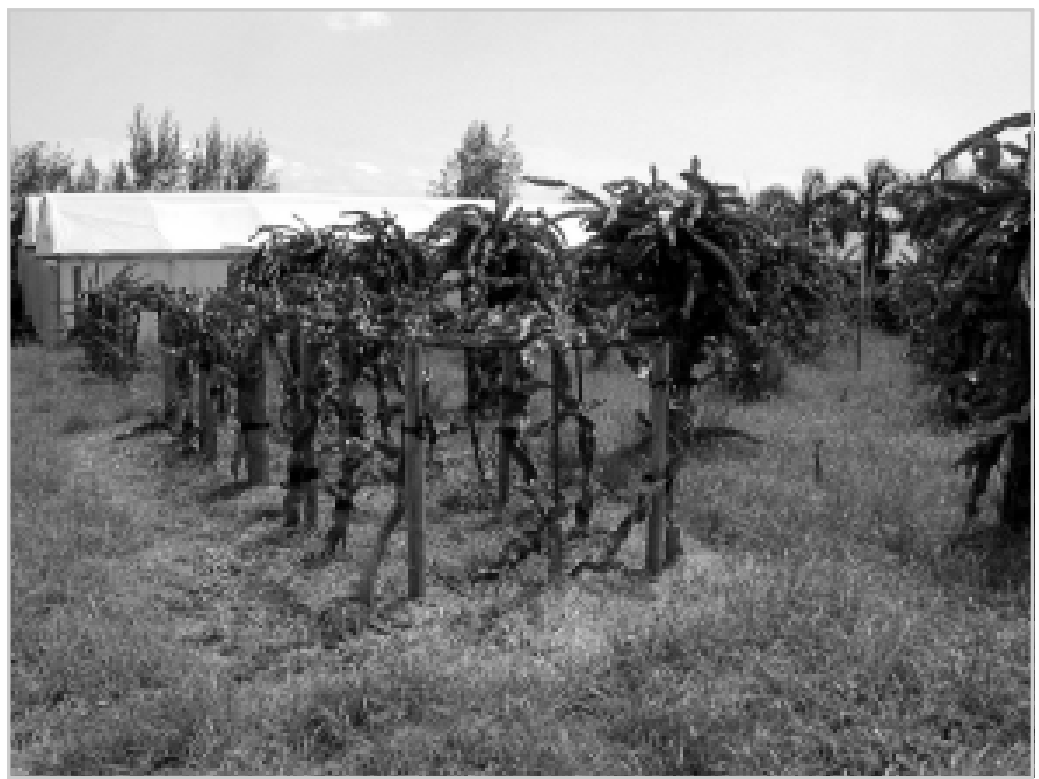

Figure 5.

Pitaya conduite sur table de production en macroparcelles expérimentales à l'île de la Réunion.

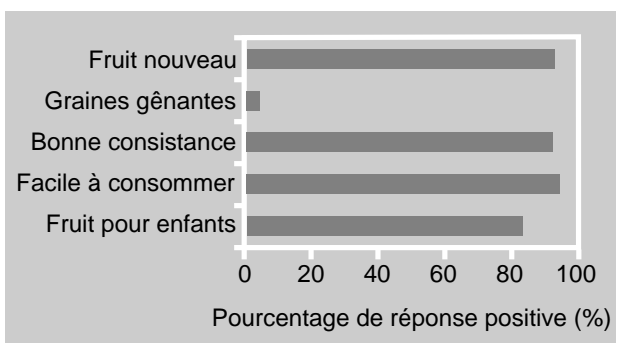

Figure 6.

Synthèse des appréciations données sur le fruit de la pitaya par un panel de 200 personnes enquêtées sur le marché forain de la ville du Tampon (île de la Réunion). Seules deux modalités ont été considérées :

[oui] ou [non + sans avis].

\section{Tableau V.}

Dépenses occasionnées par les coûts de plantation et d'entretien, chiffre d'affaire et retour sur investissements générés par 0,5 ha de pitaya conduits sur « table de production » ou sur tuteur individuel à l'île de la Réunion.

\begin{tabular}{|c|c|c|c|c|c|c|}
\hline \multirow{2}{*}{$\begin{array}{l}\text { Nombre d'années } \\
\text { à partir de } \\
\text { la plantation }\end{array}$} & \multicolumn{2}{|c|}{ Dépenses $(€)$} & \multicolumn{2}{|c|}{ Chiffre d'affaire $(€)$} & \multicolumn{2}{|c|}{ Retour d'investissement $(€)$} \\
\hline & $\begin{array}{l}\text { Conduite } \\
\text { sur tables }\end{array}$ & $\begin{array}{l}\text { Conduite } \\
\text { sur tuteurs }{ }^{2}\end{array}$ & $\begin{array}{l}\text { Conduite } \\
\text { sur tables }{ }^{1}\end{array}$ & $\begin{array}{l}\text { Conduite } \\
\text { sur tuteurs }{ }^{1}\end{array}$ & $\begin{array}{l}\text { Conduite } \\
\text { sur tables }{ }^{1}\end{array}$ & $\begin{array}{l}\text { Conduite } \\
\text { sur tuteurs }\end{array}$ \\
\hline 1 & 17,042 & 14,101 & 0 & 0 & $-17,042$ & $-14,101$ \\
\hline 2 & 3,567 & 1,372 & 10,899 & 6,288 & $-9,710$ & $-9,185$ \\
\hline 3 & 5,945 & 2,287 & 24,177 & 13,948 & 8,522 & 2,476 \\
\hline 4 & 8,323 & 3,201 & 34,680 & 20,008 & 34,879 & 19,283 \\
\hline
\end{tabular}

1 Tables de production : sur la base de 260 tables de 6,6 $\mathrm{m} \times$ par ha ; densité d'implantation des tables : $1,10 \mathrm{~m} \times 4 \mathrm{~m}$; densité : 6500 plants.ha ${ }^{-1}$.

${ }^{2}$ Tuteurs individuels : sur la base de 1250 tuteurs en pin traité par ha ; densité d'implantation des tuteurs : $2 \mathrm{~m} \times 4 \mathrm{~m} ; 3750$ plants-ha ${ }^{-1}$. 
À court terme, le marché local devrait permettre d'écouler facilement de petites quantités de fruits de l'ordre de quelques dizaines de tonnes. À moyen ou long termes, si les volumes produits venaient à augmenter, l'exportation vers l'Europe pourrait être envisagée car la période de production à l'île de la Réunion est décalée par rapport à celle des autres pays producteurs (Vietnam et Israël) et les prix pratiqués [18] restent encore favorables à un produit de qualité (marché de niche), même provenant d'une région à coût de maind'œuvre élevé.

\section{Forces et faiblesses de la culture d'Hylocereus sp. à l'île de la Réunion}

De l'ensemble de l'étude qui vient d'être présentée, il ressort que l'exploitation de la pitaya à l'île de la Réunion présente un certain nombre de points forts qui permettent de valider le choix qui a été fait de promouvoir cette espèce fruitière en culture de diversification (tableau VI). En effet, les quelques faiblesses identifiées ne suffisent pas à discréditer cette production qui procure par ailleurs des avantages indéniables à l'agriculteur et au vendeur tout en satisfaisant les goûts du consommateur.

\section{Conclusions}

Le contexte réunionnais semble favorable au développement de cultures nouvelles [5] (agro-tourisme, engouement du consommateur pour la nouveauté, soutien des collectivités locales telles que le conseil général et le conseil régional de l'île de la Réunion, image d'un "produit pays ", etc.). Un accompagnement réussi nécessite obligatoirement une approche pluridisciplinaire car il ne suffit pas de produire encore faut-il bien vendre. Ce principe a été adopté pour accompagner le développement de la culture de la pitaya dans l'île. Après avoir résolu certains problèmes techniques comme la stérilité de la plante et la mise au point de méthodes de cultures, il a fallu s'assurer de l'adhésion du consommateur à ce nouveau fruit. Cette démarche a été favorisée par les médias réunionnais (presse et télévision). Le commerçant, intermédiaire entre le consommateur et le producteur, a ensuite été sensibilisé par certains arguments comme l'élargissement de sa gamme de produit ou les qualités commerciales du fruit. Enfin, le producteur, à qui revenait la décision finale de développer ou non cette nouvelle culture, a été associé à son lancement au travers d'une adhésion volontariste ; une nouvelle filière est ainsi née.

Conscients de la jeunesse et de la fragilité de cette nouvelle filière, les producteurs

\section{Tableau VI.}

Forces et faiblesses identifiées lors d'une démarche de diversification fruitière entreprise à partir du développement de la culture de la pitaya (Hylocereus sp.) à l'île de la Réunion.

\section{Forces}

Faiblesses

\section{Esthétique et attractivité du fruit nouveau \\ Plante très rustique et facile à multiplier \\ Plante acclimatée à l'île de la Réunion \\ Entrée en production rapide \\ Retour sur investissement dès 3 ans \\ Prévision de récolte aisée \\ Production étalée sur 6 mois \\ Fruits de qualité et peu fragiles \\ Bonne aptitude à la conservation (30 j)}

Prix de vente élevé accepté par le consommateur $\left(4,57 € \cdot \mathrm{kg}^{-1}\right)$

Différentes valorisations possibles (frais, transformation, décoration)

Débouché à l'export possible (marché favorable, contre-saison)

\author{
Marché inexistant à la Réunion car fruit nouveau \\ Coût de plantation élevé car tuteurage obligatoire \\ Main-d'œuvre importante (pollinisation)
}


se sont regroupés au sein d'une association (association des Producteurs de pitaya de la Réunion ou APPIR) afin d'organiser collectivement la production et la commercialisation des pitayas. À l'avenir, cette dynamique sera soutenue et relayée par la mise en place d'un projet financé par le conseil régional de la Réunion et par l'Europe, visant à élaborer des itinéraires techniques respectueux de l'environnement ; il conviendra également d'anticiper les problèmes liés à l'intensification d'une nouvelle culture (problèmes phytosanitaires, par exemple), d'approfondir les connaissances de la plante et la caractérisation de son fruit et de favoriser sa commercialisation par la mise au point de techniques post-récolte, l'attribution d'un label, etc. Ce projet associera tous les acteurs de l'agriculture réunionnaise : chercheurs, développeurs et producteurs.

En dépit des faibles surfaces plantées aujourd'hui, les qualités de la pitaya, l'enthousiasme des consommateurs et la motivation des agriculteurs envers ce nouveau fruit font de cette espèce une véritable opportunité de diversification pour l'île de la Réunion.

\section{Références}

[1] Rivals P., Les espèces fruitières introduites à La Réunion, Imprimerie A. Gomes, Toulouse, France, 1960.

[2] Trouette E., Introduction de végétaux à la Réunion, Nouvelle Imprimerie Dionysienne (NID), Saint-Denis, France, 1898

[3] Le Bellec F., Renard V., Le grand livre des fruits tropicaux, Orphie Éditions, Saint-Denis, France, 1997, 180 p.

[4] Cadet T., La végétation de l'île de la Réunion, étude phytoécologique et phytosociologique, thèse, France, 1977.

[5] Normand F., Résultats d'une action de diversification fruitière menée à l'île de la Réunion, Fruits 54 (4) (1999) 233-245.
[6] Leakey R.R.B., Tchoundjeu Z., Diversification of tree crops: domestication of companion crops for poverty reduction and environmental services, Expl. Agric. 37 (2001) 279-296.

[7] Nerd A., Aronson J.A., Mizrahi J., Introduction and domestication of rare and wild fruit and nut trees for desert areas, in: Janick J., Simons J.E. (Eds.), Advances in new crops: proceedings of the first national symposium New crops, research, development, economics, Indianapolis, Indiana, Oct. 23-26, Portland Timber Press, USA, 1998, pp. 355-363.

[8] Wilson L.A., Agricultural diversification and processing: the way forward, Trop. Fruits Newsl. 27 (1998) 7-12.

[9] Le Bellec F., Vers un développement des espèces fruitières sous-utilisées à la Réunion, Fruits Oubliés 4 (1998) 1-3.

[10] Le Bellec F., Le patrimoine fruitier de l'île de la Réunion, Cirad-Flhor (Éd.), Saint-Pierre, France, 1993, $53 \mathrm{p}$.

[11] Nerd A., Mizrahi Y., Reproductive biology of cactus fruit crops, Hortic. Rev. 18 (1997) 321-346.

[12] Barbeau G., La pitahaya rouge, un nouveau fruit exotique, Fruits 45 (1990) 141-174.

[13] Jacobs D., Pitahaya, Hylocereus undatus, a potentiel new crop for Australia, Rare Fruit Counc. Austr. Inc. Newsl. 107 (1998) 3-7.

[14] Bosser J., Cadet Th., Gueho J., Marais W., Flore des Mascareignes, Tome 103, Famille des Cactaceae, Off. Rech. Sci. Tech. Outremer (Orstom), Paris, France, 1984, pp. 1-8.

[15] Lavergne R., Fleurs de Bourbon, Tome 7, Impr. Cazal, Saint-Denis, France, 1984, pp. 173-191.

[16] Le Bellec F., Judith R.C., Schröder A., Dijoux S., La pitahaya à l'île de la Réunion, un nouveau fruit bien séduisant, FruiTrop 41 (1997) 17-18.

[17] Raveh E., Weiss J., Nerd A., Mizrahi Y., Pitayas (genus Hylocereus) new fruit crop for the Negev Desert of Israel, in: Janick J., Simon J.E. (Eds.), New crops, Wiley, New York, USA, 1993, pp. 491-495.

[18] Imbert E., La Pitahaya, un marché en devenir, FruiTrop 80 (2001) 13. 


\section{La pitahaya (Hylocereus sp.) en cultivo de diversificación en la isla de la Reunión: estrategia de acompañamiento de su desarrollo.}

Resumen - El patrimonio frutal de la isla de la Reunión. Dentro de las aproximadamente 138 especies frutales que componen el patrimonio frutal de la isla de la Reunión, sólo algunas se cultivan verdaderamente. Con todo, otras especies podrían seducir a los consumidores amantes de novedades. Criterios de preselección para un cultivo de diversificación. Ante este hecho, se realizó una preselección con el fin de identificar especies frutales nuevas, candidatas para la diversificación en la Reunión; los criterios de elección fueron: el atractivo de la fruta, facilidad de consumo, sabor, conservación, rentabilidad de la especie, etc. Se escogió la pitahaya (Hylocereus sp.). Esta elección fue validada a continuación mediante una encuesta entre los consumidores de la Reunión. Desarrollo de la pitahaya en la isla de la Reunión. El desarrollo de este nuevo cultivo se realizó en tres etapas: definición de las dificultades agronómicas vinculadas a la intensificación de este cultivo y búsqueda de soluciones; adopción de las técnicas de cultivo básicas y sensibilización del público en general; consideración de las dificultades de los agricultores y acompañamiento comercial del nuevo producto. Conclusiones. Las calidades comerciales de la pitahaya (color y forma atractivas), el entusiasmo de los consumidores y la motivación de los agricultores hacia esta nueva especie hacen de $H$. sp. una verdadera oportunidad de diversificación para la isla de la Reunión mientras que, hace 7 años, el fruto de esta especie era completamente desconocido en la isla.

Reunión / Hylocereus / diversificación / desarrollo agrícola / manejo del cultivo / agricultores / negociantes / encuestas al consumidor

To access this journal online: www.edpsciences.org 